\title{
„Skill Mix erfordert eine klare Patientenführung“
}

\author{
Die Pflege muss in ihre neue alte Rolle hineinwachsen
}

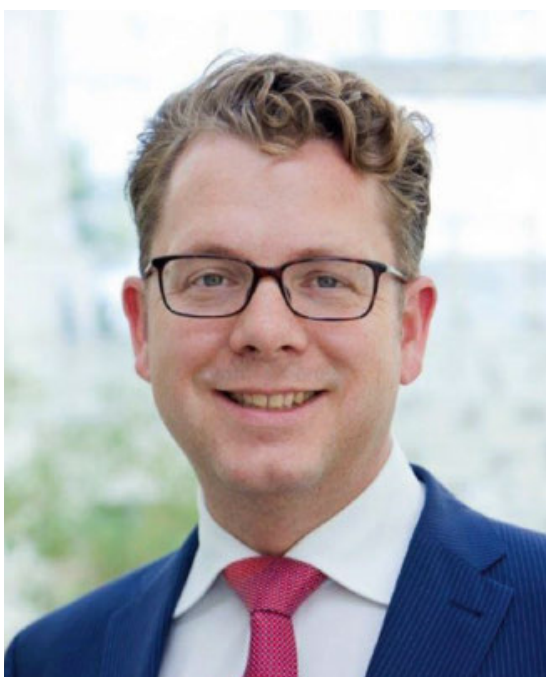

Seit Dezember 2013 führt Nicolas von Oppen, Krankenhausbetriebswirt, die Geschäfte des Klinikums Landshut. Das 520-Betten-Haus der Schwerpunktversorgung in Niederbayern wird als gemeinnützige $\mathrm{GmbH}$ in kommunaler Trägerschaft geführt. Die Fallzahlen sind in den vergangenen 4 Jahren um etwa 10000 auf 61500 Patienten gestiegen, was primär auf einen Anstieg der ambulanten Behandlungen zurückzuführen ist. Stationär werden knapp 24000 Patienten versorgt. Die Zahl der Mitarbeiter stieg um 170 auf gut 1400 Personen. Am Bett sind rund 200 Ärzte und 355 Pflegefachkräfte tätig, darunter viele mit einer Zusatzqualifikation beispielsweise im Wundmanagement, Palliative Care oder Stroke Unit. Hinzu kommen 8 Pflegefachhelfer, 6 Altenpfleger sowie je 12 Hebammen/Entbindungshelfer, Physiotherapeuten und Radiologieassistenten und 14 Laborassistenten. Psychologen, Ergotherapeuten, Logopäden, Masseure, Diätassistenten und Schmerztherapeuten mit etwa einer Stelle im Schnitt. Insgesamt beschäftigt das Klinikum 38 verschiedene Berufsgruppen, darunter auch relativ junge Berufe wie den Operationstechnischen und den Anästhesietechnischen Assistenten. Seit 2016 gehört der Gefäßassistent ebenfalls dazu. Die Einstellung eines Physician Assistant ist geplant. Über den neuen Skill Mix berichtete von Oppen auf dem Gesundheitskongress des Westens 2017.

\section{Der Fachkräftemangel in Medizin und Pflege ist in aller Munde. Wie stellt sich die personelle Situation im Klinikum Landshut dar, Herr von Oppen?}

Natürlich gibt es immer mal wieder Engpässe, insgesamt sind wir aber in der glücklichen Lage, dass wir die meisten Stellen in der Medizin und Pflege sehr gut besetzt haben. Hierzu trägt sicherlich bei, dass wir im Großraum München liegen und dadurch einen attraktiven Standort bieten. Einige Mitarbeiter pendeln täglich. Leider stellen wir aber auch fest, dass das Niveau der Bewerbungen nicht mehr das Gleiche ist wie vor einigen Jahren.

\section{Tragen die neuen Assistenzberufe dazu} bei, Ihre hohe Personaldecke zu halten? Ja und nein. Die Assistenzkräfte helfen Spitzenbelastungen zu reduzieren. Sie werden keine Fachkraftstellen ersetzen. Wir glauben vielmehr, dass sich die herkömmliche Arbeitsteilung in ärztliche und pflegerische Tätigkeiten auf den Stationen verändern muss, da ein Teil dessen, was mit den Berufsbildern traditionell verbunden wird, nicht mehr zeitgemäß ist. Statt der Pflege übernehmen heute Servicekräfte die Essensausgabe auf den Stationen und das Bettenmachen. Für die Blutentnahme haben wir einen Extradienst eingerichtet, sodass kein Arzt geholt werden muss.

\section{Was sind die Vorteile im Vergleich zur herkömmlichen Arbeitsteilung?}

Durch die Entlastung bei organisatorischen Aufgaben gewinnen Ärzte und Pfleger nicht nur mehr Zeit, ihr Pensum zu bewältigen. Sie sind auch motivierter bei der Arbeit, da sie ihrer Qualifikation entsprechend eingesetzt werden. Gleiches gilt für die Stationsund Menüassistenten, die Kodier- und andere Assistenzkräfte, die ja eigens für diese Aufgaben eingestellt wurden. Wir versprechen uns niedrige Verweildauern und ein gutes Behandlungsergebnis für die Patienten, wenn wir die Fach- und Assistenzkräfte richtig einsetzen. Derzeit bleiben die Patienten im Schnitt 6 Tage.

\section{Welche Herausforderungen sind damit} verbunden?

Durch die neuen Berufe wächst die Zahl der Personen, die rund um den Patienten tätig sind, was einen hohen Abstimmungsbedarf mit sich bringt. Die Berufsgruppen, wozu neben Ärzten, Pflegern und Assistenten auch Therapeuten und Sozialarbeiter gehören, müssen gut kommunizieren können und sich respektvoll begegnen. Der Pflege wird zudem ein hohes Koordinations- und Organisationsvermögen abverlangt. Das ist nicht einfach. Wir bieten Schulungen, externe Coachings und Projektberatung an, um die Abläufe auf den Stationen zu verbessern. Ein fortwährendes Problem sind gemeinsame Visiten auf den internistischen Stationen. Die Pflege braucht eine Terminverlässlichkeit der Ärzte, um den Tagesablauf planen zu können. Dies ist auf den internistischen Stationen ungleich schwerer als in der Chirurgie, da bei vielen Patienten die Diagnostik noch nicht abgeschlossen ist, wenn sie auf die Station kommen. Dadurch kommt es zu Verzögerungen, die an anderer Stelle kompensiert werden müssen. Unsere Mitarbeiterbefragung hat gezeigt, dass Pflegekräfte auf den chirurgischen Stationen zufriedener sind, da dort die Abläufe strukturierter sind.

\section{Sie planen einen Physician Assistant} einzustellen, der dem Ärztlichen Dienst zugeordnet ist. Auf wessen Initiative geht dies zurück?

Seit einem Jahr ist bereits ein Gefäßassistent im Ärztlichen Dienst tätig. PD Dr. Georgios Meimarakais, Chefarzt für Gefäßchirurgie, hatte sich dafür eingesetzt hat. Das Berufsbild basiert auf Empfehlungen der Deutschen Gesellschaft für Gefäßchirurgie. Von mir geht die Initiative aus, zusätzlich einen Physician Assistant einzustellen, der ein breiteres Einsatzfeld abdeckt. Das Berufsbild stammt aus den USA. Es qualifiziert für zuarbeitende, vorbereitende, unterstützende und mitwirkende Tätigkeiten, die dann eigenverantwortlich 
ausgeübt werden, beispielsweise funktionsdiagnostische Maßnahmen und Patienten- und Angehörigenberatung. Noch ist offen, in welcher chirurgischen Abteilung wir ihn einsetzen werden. Die Rückmeldungen der Chefärzte sind geteilt, einige würden lieber einen Assistenzarzt einstellen. Das heißt, ich muss noch Überzeugungsarbeit leisten, denn die Integration eines neuen Berufs kann aber nur funktionieren, wenn es gewollt ist.

\section{Sind darüber hinaus personelle Aufstockungen bei den Assistenz- berufen vorgesehen?}

Wir können uns das gut vorstellen. Sie erfahren eine hohe Akzeptanz auf den Stationen, im OP und den Funktionsbereichen. Auch der Betriebsrat und andere Gremien äußern sich positiv. Da wir für das Haus eine weitere Leistungssteigerung erwarten, werden wir insgesamt mehr Personal brauchen, also auch Assistenz. Wie sich der Bedarf konkret darstellt, muss aber in den Kliniken und Fachabteilungen beziffert werden. Im Moment sind keine weiteren Assistenzstellen eingeplant.

\section{Inwieweit werden sich Kommunika- tion, Führungsstil und Entscheidungs- strukturen im Zusammenspiel der verschiedenen Professionen am Bett verändern müssen?}

Der Skill Mix erfordert eine klare Patientenführung auf der Station. Diese Rolle sollte die Pflege einnehmen und sich mit den Ärzten hierzu abstimmen, denn die Pflege ist auf der Station präsent und kennt die Bedürfnisse der Patienten. Das Kümmern gehörte schon immer dazu, wird aber künftig - bedingt durch die kurzen Liegezeiten und den hohen Abstimmungsbedarf - noch anspruchsvoller werden. Das tradierte Bild, der Arzt entscheidet und die Pflege setzt um, hält dem nicht Stand. Im Gegenteil, um Entscheidungen patientennah auf den Stationen treffen zu können, werden flache Hierarchien, kurze Wege und gut ausgebildete Pflegefachkräfte immer wichtiger. Gefragt ist eine Kommunikation auf Augenhöhe mit Ärzten. In diese Rolle muss die Pflege aber erst noch hereinwachsen. Wir unterstützen bestehende Stationsleitungen durch Schulungsprogramme. Darüber hinaus bieten über unsere Berufsfachschule in Kooperation mit der Technischen Hochschule Deggendorf ein duales Pflege-Studi- um an, damit wir unsere Pflegenden von morgen selber ausbilden können. Wir brauchen eine selbstbewusste Pflege, die den Patienten im Stationsalltag führt.

\section{Was tut die Geschäftsführung, um diesen Prozess zu unterstützen?}

Wir stehen erst am Anfang der Entwicklung, Eine gute Kommunikation auf den Stationen braucht Vorbilder. Als Geschäftsführer muss ich eine offene Kommunikation vorleben, unterstützen, motivieren und die nötigen Freiräume schaffen, damit sich die Mitarbeiter und Führungskräfte gestaltend einbringen können. Manches ist leichter gesagt als getan. Mir geht es darum Veränderung zuzulassen, wenn nicht gar zu provozieren. In den Teams entstehen die besten Ideen, so können wir es schaffen, Organisation neu zu denken. Ein autoritärer Führungsstil würde dies unterbinden.

\section{Vielen Dank für das Gespräch, Herr von Oppen!}

Das Interview führte Dr. Adelheid Weßling, freie Journalistin, Düsseldorf.

\section{ZUR PERSON}

Nicolas von Oppen, Krankenhausbetriebswirt, startete seine berufliche Karriere 1997 als Assistenz der Verwaltungsleitung im Rotkreuzklinikum in München. Anschließend war er mehrere Jahre in 2 Kliniken der Ameos Gruppe in leitender Funktion tätig. Von 2011 bis November 2013 gehörte von Oppen dem Vorstand der Amper Klinik AG an, einer Tochter der Rhön Klinikums, bevor er im Dezember 2013 in die Position des Geschäftsführers des Klinikums Landshut wechselte.

Kontakt: vonoppen@klinikum-landshut.de 